Mauricio Sierra, Transporte neumatico de Solidos

\title{
METODOLOGIA PARA LA DETERMINACIÓN DE DIAMETRO OPTIMO ECONOMICO PARA UN SISTEMA DE TRANSPORTE NEUMATICO DE SOLIDOS EN FASE DILUIDA.
}

\section{Methodology For Determining The Optimum Economic Diameter Pneumatic Conveying Of Solids In Diluite Phase}

\author{
Mauricio J. Sierra ${ }^{1}$. Fabián A. Ortega Quitana ${ }^{2}$, Emiro A. López ${ }^{3}$, Viviana P. Villarreal ${ }^{4}$ \\ Recibido para publicación: 5 de marzo del 2013 - Aceptado para publicación: 8 de mayo del 2013
}

\section{RESUMEN}

Los sistemas de transporte neumático, presentan un uso limitado en la industria a causa del supuesto excesivo consumo de energía, agravado aún más por la ausencia de investigaciones, trabajos experimentales, análisis completos. El Objetivo general es ofrecer una metodología para determinar el diámetro económico de tubería para sistemas de transporte de sólidos de fase diluida, Metodología comprende tres (3) etapas: Establecimiento de los parámetros de, Determinación de los Costos Totales de inversión y la Obtención del modelo matemático de los costos en función del diámetro de tubería. Los Resultados obtenidos muestran un diámetro óptimo económico para el sistema de 2 pulg. , una velocidad del gas $(\mathrm{Vg})$ de 45,45 m/s, caída de presión ( P) en el sistema de 4,04 mbar/m, concentración de sólidos (\% Vs/Vg): 0,06. Conclusión, este trabajo demuestra que los costos totales de inversión en función del diámetro de tubería presentan un óptimo de 2 pulg, el modelo obtenido presenta un coeficiente de determinación R2: 1,0000 y se cumplen los parámetros de transporte neumático de sólidos en fase diluida( $\mathrm{P}, \mathrm{Vg}$ y concentración de sólidos).

Palabras claves: Evaluación económica, optimización, caída de presión.

\section{ABSTRACT}

Pneumatic conveying systems, have limited use in industry because of excessive energy consumption of course, further aggravated by the lack of research, experimental work, a complete analysis. The overall objective is to provide a methodology to determine the economic diameter of pipe for solids transport systems dilute phase, Methodology consists of three (3) stages: Establishment of parameters, Determination of total investment costs and the Recovery Model mathematical costs depending on the diameter of pipe. Results obtained show an economic optimum diameter for 2 inch system. , Gas velocity $(\mathrm{Vg})$ of $45.45 \mathrm{~m} / \mathrm{s}$, pressure drop ( $\mathrm{P}$ ) in the system of $4.04 \mathrm{mbar} / \mathrm{m}$, solid concentration (\% Vs/ Vg):0.06. Conclusion, this work shows that 
the total investment costs depending on pipe diameter have an optimal 2-inch, the model obtained has a coefficient of determination $\mathrm{R}^{2}$ : 1.0000 and meet the parameters of pneumatic transport of solids in dilute phase ( $\mathrm{P}, \mathrm{Vg}$ and concentration of solids).

Keywords: Economic evaluation, optimization, pressure drop.

\section{INTRODUCCIÓN}

Los accidentes de tránsito diariamente causan la muerte a miles de personas en todo el mundo, muertes que se pueden evitar realizando acciones para mejorar la seguridad vial[7]. Se requiere promover entre los conductores de cualquier tipo de vehículo, un cambio de conducta y una cultura preventiva, a través de espacios donde se publiquen temas relacionados con la prevención de riesgos en las carreteras. La accidentalidad en las vías se ha convertido en un problema de salud pública para la sociedad actual, según la Organización Mundial de la Salud (OMS) se tiene previsto que para el año 2030 los accidentes de tránsito sean la quinta causa de muerte de las personas a nivel mundial (Cámara de Comercio de Bogotá, n.d).

Por muchos años los gases han sido utilizados satisfactoriamente en la industria para transportar un amplio rango de sólidos particulados, desde harina de trigo a granos de trigo y de chips plásticos a carbón (2). En la actualidad existen empresas que se limitan a operar sistemas de transportes neumáticos por excesivo costo de energía que alcanza los $18,82 \mathrm{MJ} /$ ton. Las causas que originan esta dificultad son: la incorrecta selección de la velocidad del gas transportador, diámetros de tubería, la existencia de los alimentadores sinfín y la infinita variedad de características físicas y aerodinámicas de los materiales a transportar, que conducen a la inexactitud de los proyectos de las instalaciones neumáticas. (12).

El transporte neumático, por sus múltiples ventajas es uno de los más avanzados medios de manipulación de sólidos, reconociéndose como una tecnología de punta para enfrentar el desarrollo industrial y una vía efectiva para la utilización racional de los recursos energéticos en determinados sectores de la economía. Los sistemas de transporte neumático, aunque presentan indicadores económicos superiores con respecto a otros transportadores mecánicos su utilización ha estado limitada y la causa fundamental ha sido un supuesto excesivo consumo de energía, lo cual se agrava por la ausencia de investigaciones y trabajos experimentales en este aspecto, unido al análisis incompleto de las posibilidades de estos sistemas (5). Una instalación de transporte neumático consta, en líneas generales, de los siguientes elementos: Ventilador centrífugo, Sistema de carga, Ciclón, sistema de descarga, Filtro de mangas y red de tuberías de diámetro adecuado (4), siendo esta última donde se genera el mayor consumo de energía por la fricción del gas y el sólido con las paredes del ducto; por tal razón, una selección errada del diámetro de la tubería conductora convertiría el sistema de transporte en una herramienta ineficiente del manejo de sólido, de aquí la importancia por establecer metodología de evaluación que ofrezcan criterios de objetivos diseño.

\section{Diámetro óptimo de una tubería}

El tamaño más económico de una tubería de conducción es aquel para el cual la suma de los costos fijos (C Fijo inv) y los costos variables

(Cvariables inv) del sistema son los menores. (5) Los costos fijos son los costos relacionados a la inversión de capital en la instalación de la tubería, Los costos variables son aquellos relacionados a los costos de operación del sistema. El análisis que se realiza, por lo general los costos de operación incluyen solamente los costos de energía. Los costos de mantenimiento ( $\left.\mathrm{C}_{\text {Mant }}\right)$ no se pueden predecir con certeza, y no se consideran en el costo total para distintos diámetros. (5)

Ctotales de inv: $\quad C_{\text {fijos inv }}+C_{\text {variables inv }}$ (1)

$C_{\text {Fijos inv }}: C_{\text {tub }}+C_{\text {Bom. }}+C_{A c c}$ Tub $+C_{\text {inst Bom. }}+C_{\text {inst tub. }}$

(2) 
Cvariables inv $: C_{\text {explotaciòn }}+C_{\text {Mant }}$

(3)

Los costos implicados en la función anterior están referidos a diferentes bases temporales. CFijos inv, tienen un momento puntual, mientras que los $\mathrm{C}_{\text {explotaciòn }}+\mathrm{C}_{\text {Mant }}$ (fundamentalmente los costes energéticos), los cuales van a estar

presentes a lo largo de toda la vida útil de la instalación. Para poder relacionarlos estos tipos de costos, deberán referirse a una base común, un período de referencia de duración anual, de forma que habrá que tener en cuenta los costos energéticos a lo largo de todo un año y los costos de inversión amortizados (13). Para ello, utilizando el factor de amortización:

Donde $r \quad(1+r)^{T}-r$ real y $T$ el tiempo

$$
F_{\text {amort }}=\frac{(1+r)^{T} \cdot r}{(1+r)^{T}-r}
$$

de amortización en años, se tiene:

Ctotales de inv anual C $_{\text {fijos inv }}{ }^{*} F_{\text {amort }}+C_{\text {variables inv }}$ (5)

De esta manera, el coste total del sistema se divide en dos términos: por un lado, el costo amortizado de la inversión y, por otro, el de energía consumida durante un año. Ésta es la función que hay que minimizar (3).

\section{Pérdida de Presión}

Para obtener una expresión de la caída de presión total a lo largo de una tubería de transporte neumático plantaremos la ecuación de cantidad de movimiento para un tramo de la misma. Considere un tramo de tubería de sección transversal $A$ y longitud $\delta L$ inclinado respecto de la horizontal un ángulo $\theta$, que transporta una suspensión de porosidad $\varepsilon$, Figura 1.

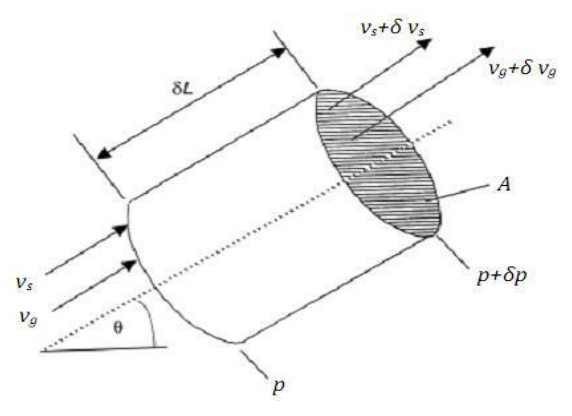

Figura 1. Tramo de tubería inclinado $\theta$ grados Para la evaluación de la perdida de presión del fluido bifasico ( Sólido+gas), debemos plantear el balance de la cantidad de movimiento (10).

(6)

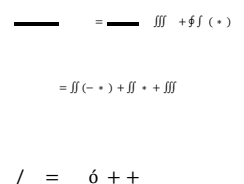

(9)

Reorganizando la ecuación (10), asumiendo que la porosidad del lecho $(\varepsilon)$, densidad del gas $(\rho g)$ permanecen constantes y dividiendo por Área transversal de la tubería tenemos:

\section{Transporte Neumático de Fase Diluida y Fase Densa}

Las publicaciones existentes de transporte neumático exhiben descripciones generales de trabajo como las citadas en la tabla 1, el límite entre el transporte en fase densa y fase diluida, sin embargo, no es marcado y aún no hay una definición universalmente aceptada de ambos tipos de transporte (9).

Tabla1. Criterios de Fase diluida y Densa

\begin{tabular}{|l|c|c|}
\hline Criterio & Fase Diluida & Fase Densa \\
\hline $\begin{array}{l}\text { Velocidad } \\
\text { gas }\end{array}$ & $\mathrm{Vg}>20 \mathrm{~m} / \mathrm{s}$ & $\mathrm{Vg}<5 \mathrm{~m} / \mathrm{s}$ \\
\hline $\begin{array}{l}\text { Concentració } \\
\mathrm{n} \text { del Sólido } \\
\% \text { v/v }\end{array}$ & $\begin{array}{c}\text { Concentració } \\
\mathrm{n}<1\end{array}$ & $\begin{array}{c}\text { Concentració } \\
\mathrm{n}>30\end{array}$ \\
\hline $\begin{array}{l}\text { Caída de } \\
\text { Presión }\end{array}$ & $<$, & $>20$, \\
\hline
\end{tabular}


\begin{tabular}{|l|l|l|}
\hline Capacidad & $<10000 \mathrm{~kg} / \mathrm{h}$ & $>10000 \mathrm{~kg} / \mathrm{h}$ \\
\hline
\end{tabular} Fuente: Rhodes 2008.

Una de las maneras de establecer un límite de diferenciación entre la fase diluida y densa en sistemas de transporte neumática es mediante el diagrama de estado, Caída de presión por unidad de longitud de tubería en función de la velocidad del gas de transporte, $\mathrm{Vg}$, para curvas de flujo de sólidos constante, Ws, como parámetro (9).

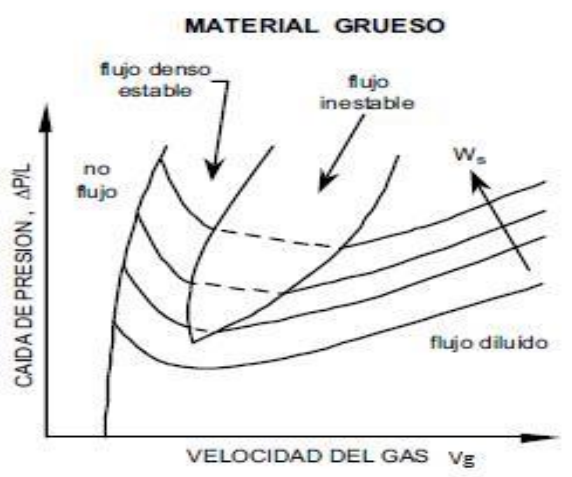

Figura 2. Diagrama de estado, en sistemas transporte neumático

\section{Materiales y Métodos}

La evaluación del diámetro optimo, corresponde al sistema de transporte neumático diseñado y construido durante el desarrollo de la asignatura de Operaciones Unitaria I del programa de ingeniera de Alimentos de la universidad de Córdoba en segundo semestre de 2010, con capacidad de $150 \mathrm{~kg} / \mathrm{h}$ en fase diluida.

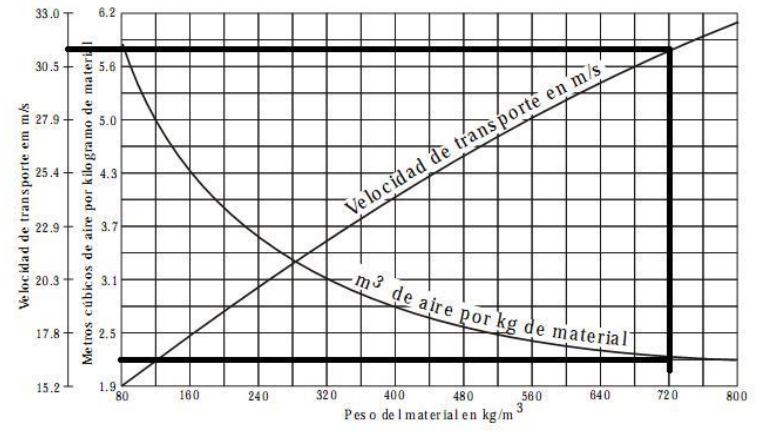

Figura 3. Sistema de transporte Neumático

\section{Establecimiento de los parámetros de diseño}

Los parámetros establecidos para el desarrollo del diseño del sistema de transporte neumático de fase diluida a nivel de laboratorio de corresponde a: Flujo de sólidos, $150 \mathrm{~kg} / \mathrm{h} ; \Delta \mathrm{P} / \mathrm{L}$ $<5 \mathrm{mbar} / \mathrm{m}$ y concentración de sólido $\mathrm{v} / \mathrm{v}<1$ $\%$. Las características del sólido Angulo de fricción interna, ángulo de deslizamiento, densidad aparente y porosidad de yuca seca al $4 \%$, correspondiente a un tamaño de partícula malla 35 (tabla 2).

\section{Determinación Costos Totales de instalación}

Para el establecimiento de los costos totales de inversión del sistema neumático, se determinaron los costos fijos de instalación de los diámetros comerciales de 1 a 4 pulg. y se estimo el consumo de energía por la potencia desarrollada por el ventilador en la geometría del sistema de la figura3.

Tabla2. Propiedades de masa/partícula del sólido

\section{Resultados y Discusión \\ Evaluación de la relación másica Sólido /gas \\ La relación solido/gas se efectuó a través del}

\begin{tabular}{|c|c|}
\hline Propiedad & Valor \\
\hline Angulo de fricción interna & $35^{\circ}$ \\
\hline Angulo de deslizamiento & $45^{\circ}$ \\
\hline Porosidad & 0,9945 \\
\hline Densidad Aparente $\left(\mathrm{g} / \mathrm{m}^{3}\right)$ & 720 \\
\hline Diámetro equivalente $(\mathrm{m})$ & 0,004 \\
\hline
\end{tabular}

nomograma, figura 4, de la empresa ventilación y representaciones industriales de la ciudad de Monterey, México. 


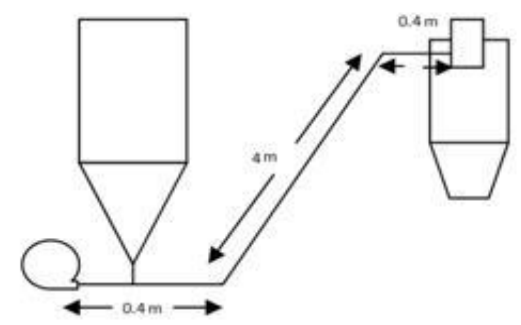

Figura 4. Nomograma de velocidad de suspensión sólidos en sistemas de transporte neumático (4).

Utilizando la figura 4 y la densidad aparente de sólido, interceptamos la curva inferior y superior; la primera nos relaciona los metros cúbicos que son necesario para suspender un kilogramo de sólido, para nuestro caso corresponde a 2,2 $\mathrm{m}^{3}$ aire/ $\mathrm{Kg}$ sólido, La curva superior nos ofrece la velocidad del gas recomendada para garantizar el transporte del sólido, $31,5 \mathrm{~m} / \mathrm{s}$ aproximadamente, este valor no necesariamente corresponde al de diseño; con la relación volumen de gas /masa de sólido podemos conocer caudal de gas requerido para el sistema de transporte neumático y relación másica sólido/gas. Los procedimientos de cálculo se desarrollaran en hojas de cálculo de

\section{Obtención de Modelo Matemático.}

La obtención del modelo matemático en función del diámetro de tubería, consiste en graficar los costos totales de instalación por diámetro que lo genero, desde 1 a 4 pulgadas. Las referencias diámetros comerciales de tubería plástica de PVC 1,1 1/4, 1 11/2, 2, 2 1/4, 2 $1 / 2,3,3 \frac{1}{2}$ y 4 .

\section{Evaluación de pérdida de Carga en Tubería}

Para la determinación de la pérdida de presión se empleo la ecuación (10), (la porosidad relaciona corresponde a la del lecho expandido al $100 \%$ (1)); para cada diámetro de evaluación. Cada valor de $\Delta \mathrm{P}$ permite determinar el consumo de energía a partir de ecuación de potencia de elementos impulsores de fluidos, multiplicada por número de horas de trabajo al año, y por el costo promedio de $\mathrm{KWh}$.

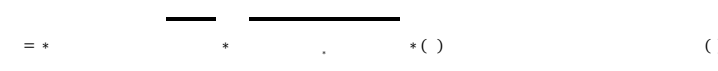

Costos Totales de Instalación Vs Diametro de Tuberia

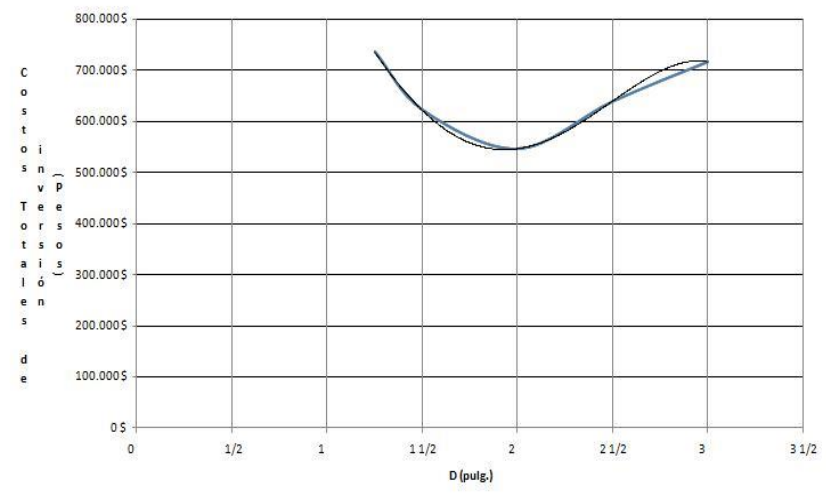

Figura 8. Costos Totales Vs Diámetro tubería

Para el caso del establecimiento de los costos de inversión en tuberías y motor para el ventilador se tomaron de los catálogos de venta 2010 de P.V.C-Gerfor S.A y Siemens C.I.A citados en las referencias (8) y (7) respectivamente.

Modelo Matemático de Costos Totales de Inversión

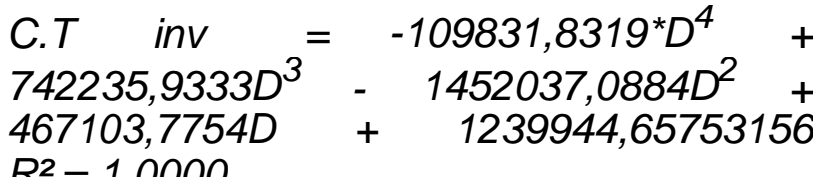
$R^{2}=1,0000$

Obtenido el modelo matemático de los costos en función del diámetro de tubería, se procede a Igualar a cero la primera 
derivada ( $\mathrm{dCT} / \mathrm{Dd}=0)$, determinando el diámetro que genera dicha igualdad, concepto conocido como Diámetro Optimo de Económico; para el sistema propuesto corresponde a 2 pulg. , el procedimiento abordado para el cálculo de esta variable se realizó a través del complemento de Excel 2007, Solver (11)

\section{CONCLUSIÓNES}

La metodología equivalente empleada, demuestra que la evaluación del diámetro optimo económica de tubería para un fluido bifásico sólido/gas y el modelo encontrado de los costos totales de inversión en función del diámetro de tubería, muestran el mismo comportamiento que a los que exhiben los fluidos newtonianos y no newtonianos, el un coeficiente de determinación $R^{2}$ : 1,0000 nos indica que las variables seleccionadas en la evaluación explican el fenómeno; los resultados obtenidos: $\Delta \mathrm{P}, \quad \mathrm{Vg} \quad \mathrm{y}$ concentración de sólidos cumplen con los criterios de transporte neumático de sólidos en fase diluida.

\section{REFERENCIAS}

[1]. Arboleda J. 2000. Teoría y práctica de la purificación del agua. McGrawHill. Santa fe de Bogotá, 413-42

[2]. Cabrejos, F; Jofré M; Rojas J. 2004. Transporte neumáticos de Materiales sólidos a granel. Universidad Técnica Federico Santa María. Chile. Congreso CONAMET/SAM, 1-6.ç

[3]. Fuentes M, Pérez R, Martínez J, López A. 2006. Optimización del sistema formado por la estación de bombeo, la tubería de impulsión y el depósito de regulación. VI
SEREA-Seminário Iberoamericano sobre Sistemas de Abastecimento Urbano de Água. João Pessoa (Brasil), 5 a 7 de junho de 2006, 1-13.

[4]. GruberHermanos. 2004. Transporte Neumático.http://www.gruberhermanos.com .[ citado 20 de noviembre de 2010.

[5]. Holzapfel E. 2003. Diámetro óptimo de una tubería. $\quad$ http:// www2.udec.cl/ riego/Publicaciones/Diámetr oTubería.[ 12 de noviembre de 2010 ].

[6]. Lesme R, Lesme N, Roca G. 2001. Economía de los sistemas de transporte neumático para bagazo de caña de azúcar. Universidad de Oriente. Cuba. Rev.: Centro Azúcar. Vol. (4):1, 1-5.

[7]. Lista de precios de Colombia. 2010. http://www.siemens.com.co.[2 de noviembre de 2010].

[8]. Lista de precios de construcción-P.V.CGerfor S.A. 2010. http://www.coval.com.co.[2 de noviembre de 2010].

[9]. Rhodes M. 2008. Introduction to particle technology", Jon Wiley \& Sons, 2nd Edition, 46-52. 